\title{
Editorial
}

\section{THE VALUE OF A MULTIDISCIPLINARY APPROACH IN ENDOCRINE SURGERY}

\begin{abstract}
The increasing complexity of medicine has led to the development of an integrated approach between nursing, surgical, medical and diagnostic experts in order to optimize patient care. These multidisciplinary care teams attempt to ensure that all patients receive coordinated attention from a group of skilled professionals, as they progress through their different phases of medical care. We believe that the underlying complexity of endocrine surgery patients coupled with the benefit of having time to pursue comprehensive evaluation and the subtle balance or risks vs benefits in our field, naturally lends itself well to an integrated approach. Although the data on the clear cut benefits of multidisciplinary care are still evolving; in this article, we delineate why we feel endocrine surgery patients benefit from a multidisciplinary approach and why we owe it to our patients to participate in and develop a multidisciplinary care structure to improve outcomes and health care system efficiency.
\end{abstract}

Keywords: Multidisciplinary care, Cancer care team, Tumor board, Cancer conference, Multidisciplinary care team.

\section{Introduction}

The increasing complexity of management decisions for cancer patients has led to the development of an integrated approach for nursing, surgical, medical and diagnostic

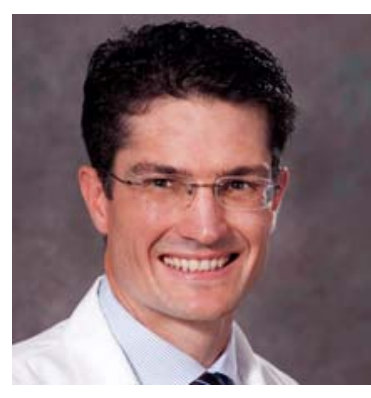

Michael Campbell

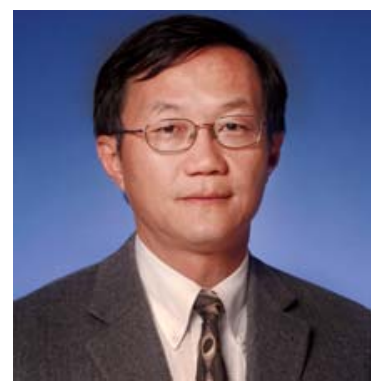

Quan-Yang Duh experts in order to optimize patient care. These multidisciplinary care teams (MDTs) attempt to ensure that all patients receive coordinated attention from a group of skilled professionals that can weigh multiple therapeutic options and remain in good communication as patients progress through their different phases of medical care. Although, there is no single model for MDTs, most MDTs aim to coordinate clinic schedules of providers with multiple specialties allowing for patient convenience and improved physician communication. A second element of the MDTs is to conduct cancer conferences or tumor boards, in which health care providers of different specialties review and discuss a patient's treatment. MDTs are thought to improve communication, coordination and decision-making among health care professionals. ${ }^{1}$

\section{Clinical Evidence for Multidisciplinary Care}

The clinical evidence supporting the use of MDTs is still evolving. Several small studies suggest that MDTs can bring about improvements in oncology care by team decision-making and increased adherence to clinical practice guidelines. ${ }^{2}$ The presentation at a multidisciplinary cancer conference has been shown to increase the percentage of patients receiving complete staging, multidisciplinary evaluation and adherence to nationally accepted care guidelines while decreasing the interval from diagnosis to treatment. ${ }^{3}$ Women in a multidisciplinary breast cancer clinic reported higher levels of satisfaction with physician and nursing care than those who received individual hospital consultation. ${ }^{4}$ Several retrospective studies have suggested that MDTs improve survival in patients with colorectal, esophageal and head and neck cancer. ${ }^{5}$ Interestingly, there is also evidence that MDTs improve the well-being of the team members and provide increased job satisfaction for physicians who participate in them. ${ }^{5}$

\section{Examples of Multidisciplinary Care in Endocrine Surgery}

While there have been no studies evaluating the benefits of MDTs in patients with surgical endocrine disease, logically there are three major reasons why endocrine surgery patients lend themselves to an integrated approach. First, endocrine disorders are frequently complex. A PubMed search for the phrase 'thyroid nodule' shows 371 publications in the English language in the last 12 months. The articles are evenly distributed over the surgical, radiology, pathology and medical endocrinology literature. Needless to say, there is a dizzying 
amount of data that are applicable to the endocrine patient. Endocrine patients require expertize from multiple specialties to diagnose, localize, prepare, operate on and assure a smooth convalesces. We recently reported a case of 36-year-old man with difficulty to control hypertension, who was found by his endocrinologist to have hyperaldos-teronism. Computed tomography of the abdomen demonstrated a $0.9 \mathrm{~cm}$ nodule in the right adrenal gland. Ten days later, he developed back pain and was taken to the operating room for repair of a Stanford type A aortic dissection. Postoperatively, he continued to have elevated blood pressures and we were consulted to; consider urgent right adrenalectomy. His case was discussed at our multidisciplinary adrenal tumor board where in reviewing his imaging and discussing his situation with our colleagues, we weighed the value of pursuing an adrenalectomy so soon after major cardiac surgery. Additionally, we contemplated the benefits of pursuing of adrenal vein sampling (AVS). Ultimately, he did undergo AVS which suggested bilateral adrenal hyperplasia and he was managed nonoperatively. In his case, it took coordinated care between two surgical teams (cardiac and endocrine), endocrinologists, radiologist, pathologists, anesthesiologists, critical care physicians and an interventional radiologist to orchestrate a successful outcome.

Another important point is that with most endocrine surgery patients, there is no urgency for an operation. With the patients we see we have the luxury of pursing further workup and involving our colleagues to coordinate their care. For example, we were referred a 52-year-old woman with primary hyperparathyroidism. A sestamibi scan did not demonstrate any abnormal uptake. An ultrasound revealed a single suspicion lesion deep to the left lobe of the thyroid. During her initial exploration, no parathyroid adenoma was found at the site suggested by the ultrasound, so a four gland exploration was performed. Eventually, right upper and left lower parathyroid adenomas were identified and excised. Biopsy of her right lower and left upper glands suggested normal parathyroid tissue. No intraoperative parathyroid hormone (PTH) testing was available, but postoperatively she continued to have an elevated calcium with a nonsuppressed PTH. Reevaluation with a sestamibi scan demonstrated subtle uptake inferior to the left submandibular gland. This was confirmed to be an ectopic parathyroid adenoma when ultrasound-guided FNA demonstrated an elevated PTH aspirate. Subsequently, she underwent a focused parathyroidectomy with intraoperative ultrasound guidance to remove this ectopic adenoma. Postoperatively, her serum calcium and PTH normalized. As with many endocrine surgery patients, in this case we had the luxury of time to sort through her complexities. This allowed us to involve a multidisciplinary team that included a diagnostic radiologist who adeptly picked up the subtle focus of an ectopic parathyroid gland on a sestamibi scan, an interventional radiologist who confirmed its presence with an aspirate and an ultrastenographer who was able to guide us to the site of the unusual gland intraoperatively.

Finally, for many of our patients the balance or risks vs benefits is not always obvious. Unlike other surgical specialties, in endocrine surgery there are often nonsurgical alternatives that we can offer in lieu of an operation. A multidisciplinary approach allows us to evaluate these options and tailor the risks and benefits to the individual patient. Let us take the example of a 68-year-old gentleman referred to us for a recurrent papillary thyroid cancer. This patient had a total thyroidectomy with bilateral central and left lateral neck dissection for node positive, multifocal tumors followed by radioactive iodine in 2005. The next year, he had a recurrence in his left neck with a redo left neck dissection and another course of radioactive iodine. In 2009, he developed another recurrence in the left neck and again underwent a reoperative neck dissection. In 2011, his thyroglobulin started rising and repeated ultrasound showed adenopathy in the right lateral and central neck that was biopsied to have metastatic papillary cancer. He underwent a direct laryngoscopy which showed normal movement of his true chords bilaterally. He remained totally asymptomatic. We discussed him at our multidisciplinary cancer conference and ultimately he underwent a reoperative right lateral and central neck dissection guided by intraoperative ultrasonography and blue-dye injection. He continues to do well. Was surgery the correct choice? In hindsight it probably was the correct decision. But what if he ended up with a permanent hypoparathyroidism? Would we feel as proud of ourselves? My guess is not. The risk to benefit ratio was a challenge to interpret in his case. Having a team of physicians managing a complex patient helps to balance that decision and maximize the benefit to the patient.

\section{Challenges to Multidisciplinary Care}

The multidisciplinary approach to endocrine care has numerous benefits, but as with all elements of health care it faces challenges. A systematic review by Lamb et al ${ }^{1}$ looking at factors that effect the decision-making 
of MDTs involved in cancer care found that time pressure, excessive caseload, low attendance, poor team working and lack of leadership lead to a deterioration of decision-making. In this study, discussion among an MDT failed to reach a decision in up to $52 \%$ of cases and the decisions made by the team could not be implemented in up to $16 \%$ of the cases. ${ }^{1}$ We have found that problems arise when there is a lack of strong leadership focusing the team. Additionally, we sometimes experience conflicting views from specialists with overlapping areas of expertize, such as the surgeon's role as a cognitician as oppose to solely a technician. Sometimes, there are different perspectives from different team members with regards to the long- and shortterm goals of the patients, and often the relative acceptance of surgical vs nonsurgical complications is weighed differently. Ultimately, despite its shortcomings a multidisciplinary approach provides mechanism in which safe, well-coordinated, data driven care can be delivered to the benefit of the patient.

\section{CONCLUSION}

Multidisciplinary care is the cornerstone to effectively caring for patients with endocrine surgical disorders. MDTs provide a mechanism for clear communication and coordinated medical care for this complex group of patients. Although data on the clear cut benefits of MDTs for endocrine surgical diseases are still evolving, we owe it to our patients to participate in and develop our multidisciplinary care structure to improve patient outcomes and health care system efficiency.

\section{REFERENCES}

1. Lamb BE, Brown KF, Nagpal K, et al. Quality of care management decisions by mulidisciplinary cancer teams: a systematic review. Ann Surg Oncol 2011;18:2116-2125.

2. Lamb BW, Sevdalis N, Mostadfid H, et al. Quality improvement in multidisciplinary cancer teams: an investigation of teamwork and clinical decision-making and cross-validation of assessments. Ann Surg Oncol 2011;18:3535-3543.

3. Freeman RK, Van Woerkom JM, Vyverberg A, et al. The effect of a multidisciplinary thoracic malignancy conference on the treatment of patients with esophageal cancer. Ann Thorac Surg 2011 Oct;92(4):1239-1242.

4. Frost MH, Arvizu RD, Jayakumar S, et al. A multidisciplinary healthcare delivery model for women with breast cancer: patient satisfaction and physical and psychosocial adjustment. Oncol Nurs Forum 1999 Nov-Dec;26(10):1673-1680.

5. Taylor C, Munro AJ, Glynne-Jones R, et al. Multidisciplinary team working in cancer: what is the evidence? BMJ 2010 Mar; 23:340.

Michael Campbell

Clinical Instructor, Department of Endocrine Surgery University of California, San Francisco, California, USA

Quan-Yang Duh

Professor, Department of Endocrine Surgery University of California, San Francisco 1600 Divisadero Street, 3rd Floor Hellman Bldg San Francisco, California-94115, USA

Phone: 415-333-7789, Fax: 415-885-7617 e-mail: quan-yang.duh@ucsfmedctr.org 